\title{
BEDTEW/RGSSERTA
}

\section{Ruolo ed organizzazione della microbiologia clinica di domani}

\section{Giorgio Mucignat', Marina Crovatto}

'Struttura Operativa Semplice di Microbiologia Clinica e T.A. ${ }^{2}$ Struttura Operativa Semplice di Immunologia C. e Virologia

Dipartimento di Medicina di Laboratorio dell'Azienda Ospedaliera “Santa Maria degli Angeli" Pordenone

Key words: Infectious diseases, microbiology service, public health

Role and Organization of Clinical Microbiology: a project for the future.

\section{SUMMARY}

Infectious diseases continue to represent an important preventive and clinical problem especially in the light of the emergence of new pathogens, of the return of infrequent pathogens and of the increased risk of infective pathologies associated with migratory flow.

Another critical point is represented by the possible insurgence of infections due to agents used in bioterrorism. More than ever it is indispensable for our country to define a network of structures able to give an organized response to these relevant problems both in terms of prevention and assistance. It is therefore important to define a protocol for clinical microbiology on a national basis that responds to different levels of activity.

Keeping in mind that the fundamental objectives of microbiology are:

- diagnosis of infective diseases

- determination of resistance to antimicrobic drugs as a valid instrument to allow the clinician to proceed with specific treatment

- control of infection spreading in the sanitary structures (hospital infections)

- to develop front line defence in new infections and bioterrorism, in collaboration with the department of prevention

- to be a reference centre for specific training

The principals on which one must base the reorganization are essentially three:

I. to configurate the microbiology service on the basis of real needs of the local population

2. to give qualitatively optimal results in real time

3. supporting "good clinical practice" to assure adequate patient results and acceptable costs for the regionale sanitary system (SSR)

The organization of microbiology should therefore foresee a microbiology department, structured on a provincial basis or a vast area including a structured centralized complex with peripheral structures (even simplified).

This must integrate actively with the department of prevention in regard to the dynamics of infectious diseases in the territory and with the Division of Infectious Diseases (where this exists).

In parallel an active interaction with research structures (IRCCS and University) present in the territory to enable the coordinated implementation of the guidelines of research and its relapse on diagnostic exams.

Keeping in mind that the quality of diagnostic performance is closely linked to the professional experience of the operators it is good practice centralizing some diagnostic procedures by identifying a reference centre.

In a regional network organized in this way it is important to include a coordinating structure, since it is necessary to activate a complete level of communication, information exchange, activity planning, and complete problem solving.

\section{INTRODUZIONE}

Le innovazioni tecnologiche, il profilarsi sempre più evidente di quella che diverrà un'emergenza infettiva per le nostre popolazioni, la globalizzazione e l'impatto importante dell'antropizzazione sulla biosfera, stanno cambiando profondamente lo scenario in cui si trovano a lavorare i microbiologi e comportando modifiche tali da porre seriamente il problema di prepararsi ed attrezzar- si per fronteggiare vecchie e nuove infezioni. J. Van Elder in occasione del Congresso ESCMID del 2000 (29) afferma che "questo è un tempo di rapidi e fondamentali cambiamenti in molti campi della gestione delle malattie infettive" e sottolinea come la Microbiologia Clinica abbia un ruolo fondamentale nella gestione delle infezioni, ma si pone anche una domanda: $i$ microbiologi sono in grado di rispondere prontamente ai cam- 
biamenti, in maniera attiva, interpretandoli ed orientandoli, oppure si limitano a subirli?

Sulla base di queste osservazioni ci siamo proposti di fare un'analisi critica dei fattori che condizionano la necessità di "aggiornare" la Microbiologia e di esprimere il nostro punto di vista su quale potrebbe essere, nell'immediato futuro, una sua razionale organizzazione su base regionale. A tal fine abbiamo fatto anche riferimento ad un documento, unico nel suo genere, del Ministero della Salute del Regno Unito, che, oltre ad una esauriente disanima delle possibili emergenze sanitarie future, analizza i risvolti di tipo organizzativo ad esse conseguenti (2).

\section{Modifiche ambientali e cambiamenti nell'utilizzo del territorio}

L'uso intensivo del territorio, reso necessario dallo sviluppo dell'agricoltura, degli allevamenti, delle attività minerarie e dalla deforestazione di aree selvagge, ha determinato un inserimento di attività umane in luoghi prima inaccessibili. Questo ha comportato un maggior rischio di esposizione ad infezioni veicolate da insetti o da altri animali e di contatto con agenti infettivi precedentemente ignoti. Un esempio è la febbre emorragica causata dal virus Ebola, identificato nel 1976 nella Repubblica Democratica del Congo, che continua tuttora a dimostrare i suoi effetti devastanti in alcune aree dell'Africa. Altro esempio è il virus della febbre di Lassa, il cui serbatoio è un topino molto comune nelle savane e foreste dell'Africa Centrale, Orientale ed Occidentale: il contagio avviene per contatto con le sue feci.

È possibile anche il contagio interumano, per contatto diretto con il sangue, secrezioni faringee, urine o tramite rapporti sessuali. In Europa sono stati già segnalati casi da importazione (12). Anche nelle nostre latitudini l'utilizzo intensivo del territorio ha determinato la disseminazione di infezioni importanti: ad esempio nelle zone nord della Gran Bretagna vengono registrate, soprattutto in primavera, epidemie di Cryptosporidium causate dalla contaminazione di acqua potabile con liquami di origine animale provenienti da allevamenti nelle zone circostanti (5).

Aumento dello spostamento di merci e persone Nel corso degli ultimi anni si è osservato un aumento esponenziale non solo della quantità ma anche della velocità degli spostamenti di merci e persone. Questo significa che se un soggetto ha un'infezione in fase di incubazione al momento in cui parte, questa si manifesta dopo l'arrivo a destinazione, spesso in un altro paese. Si stima che circa due milioni di persone attraversino le frontiere ogni giorno nel mondo e che per un milione di esse il passaggio avviene da un paese in via di sviluppo ad uno sviluppato, con tutte le ovvie conseguenze. Si sta osservando un aumento dei viaggi verso mete diverse, quali le località esotiche ed a questo corrisponde un aumento di forme gravi di malaria da Plasmodium falciparum e di casi di malaria cerebrale.

\section{Cambiamenti climatici}

Secondo un rapporto del Ministero della salute inglese (Health Effects of Climate Change: an export review for comment. London: Department of Health, 2001) i cambiamenti climatici più immediati provocheranno un riscaldamento complessivo della crosta terreste con inverni meno freddi e temperatura più elevata delle acque.

Le possibili conseguenze di queste modifiche saranno:

- Incremento della sopravvivenza dei patogeni nelle acque naturali;

- Calo della qualità delle acque potabili;

- Aumento dell'utilizzo di aria condizionata con aumento della diffusione di Legionella pneumophila;

- Aumento delle popolazioni di alghe;

- Aumento degli eventi atmosferici avversi e delle inondazioni con conseguenti effetti negativi sulla potabilità stessa delle acque;

- Aumento del contatto umano con le acque dolci e marine;

- Aumento delle tossinfezioni alimentari;

- Aumento del colera e delle febbri tifoidi;

- Maggiore disponibilità di habitat per alcuni insetti quali l'anofele e conseguente aumento dei casi di malaria;

\section{Comportamenti umani a rischio}

La lenta ma continua liberalizzazione dei costumi sessuali, associata ad uno scarso utilizzo di protezioni durante l'atto sessuale sta determinando, soprattutto tra gli adolescenti inglesi, ma non solo, un aumento di malattie sessuali quali ad esempio la sifilide. La serietà del problema è enfatizzata dalla possibilità di trasmissione associata dell'HIV. L'uso promiscuo di siringhe tra $\mathrm{i}$ tossicodipendenti mantiene o addirittura fa aumentare le infezioni da virus veicolate dal sangue quali HIV e virus epatitici (HBV, HCV), favorite anche dall'aumento indiscriminato dei tatuaggi $(30,16)$.

\section{Emergenze dovute a crisi dei sistemi di controllo} Sono state osservate emergenze infettive dipendenti dal venir meno di condizioni di controllo istituzionali per le cause più svariate. Un esempio legato ad una crisi politico-istituzionale è l'aumento dei casi di difterite che si sono verificati in 
Unione Sovietica nel 1995 come conseguenza della crisi di stato. Un altro esempio è legato alla pertosse: una diminuzione della popolazione vaccinata dovuta alle allarmanti notizie sulle reazioni avverse del vaccino ha causato, in Gran Bretagna, dal 1975 al 1990 un ritorno della malattia a livelli quasi sovrapponibili a quelli presenti prima della campagna vaccinale (18).

Adattamento dei microrganismi agli antibiotici I microrganismi sono in grado di adattarsi velocemente alle condizioni ad essi sfavorevoli quali possono essere $\mathrm{i}$ trattamenti antibiotici e anche modifiche genetiche casuali possono diventare importanti per la sopravvivenza: è il caso della meticillino-resistenza di Staphylococcus aureus (MRSA). Negli ultimi anni si è registrato un aumento di presenza di MRSA nelle emocolture: in Gran Bretagna si sta avvicinando al 50\%. Il fenomeno delle resistenze non è certo limitato solo agli stafilococchi e alla meticillina, come si può chiaramente evincere dalla tabella 1 , stilata dalla WHO già nel 1999.

\section{Tabella I.}

\begin{tabular}{lll}
\hline PATOLOGIA & MICRORGANISMO & RESISTENZA \\
\hline Dissenteria & Shigella dysenteria & Multiresistenza \\
\hline Gonorrea & Neisseria gonorrhoeae & Penicillina e \\
& & Tetraciclina \\
\hline Infezioni & Enterococcus spp. & Vancomicina \\
nosocomiali & Klebsiella spp. & Multiresistenza \\
& Pseudomonas spp. & Multiresistenza \\
& Staphylococcus aureus & Meticillina \\
\hline Polmonite & Streptococcus pneumoniae & Multiresistenza \\
\hline Febbri tifoidi & Salmonella enterica & Multiresistenza \\
\hline
\end{tabular}

\section{Deficit del sistema immunitario}

Patologie nuove quali quelle da retrovirus (in particolare l'AIDS), le malattie autoimmuni, il diabete mellito, i progressi della medicina nel campo dei trapianti e della terapie antineoplastiche, hanno comportato un aumento dei pazienti immunocompromessi.

Altre situazioni di stress immunologico sono legate ai cambiamenti nella gestione del paziente: interventi chirurgici importanti, uso di cateteri e tubi per la ventilazione, lungodegenza dei pazienti immunocompromessi.

Anche l'allungamento della vita è associato ad una diminuzione dell'attività immunitaria e dal parallelo aumento dei rischi infettivi, così come accade anche nei nati prematuri.

Tutti questi pazienti vengono aggrediti anche da microrganismi (opportunisti) che nel soggetto normale non sono in grado di dare malattia e che richiedono trattamenti specifici. Ne consegue un maggior consumo di antibiotici ed un aumento di possibilità di selezionare dei ceppi resistenti.
Vecchi e nuovi focolai di epidemie ed endemie Le malattie infettive classiche (TBC, Malaria ecc.) sono ben lontane dall'essere sconfitte. Le condizioni igieniche precarie dei paesi in via di sviluppo favoriscono la loro persistenza ed addirittura in alcune aree c'è un aumento della loro incidenza e la minaccia di agenti infettivi nuovi o sconosciuti è sempre presente. Basti pensare che dagli anni settanta sono stati scoperti almeno trenta nuovi patogeni. Alcuni esempi si possono vedere nella tabella stilata dalla WHO, aggiornata al 1999.

Tabella 2. Newly identified infectious diseases and pathogens: some examples

\begin{tabular}{ll}
\hline 1999 & Nipah virus \\
\hline 1997 & H5NI avian flu \\
\hline 1996 & $\begin{array}{l}\text { nvCJD } \\
\text { Australian bat lyssavirus }\end{array}$ \\
\hline 1995 & HHV8 (Kaposi sarcoma virus) \\
\hline 1994 & $\begin{array}{l}\text { Sabia virus } \\
\text { Hendra virus }\end{array}$ \\
\hline 1993 & $\begin{array}{l}\text { Hantavirus polmonary sindrome } \\
\text { (Sin Nombre virus) }\end{array}$ \\
\hline 1992 & Vibrio cholerae 0I39 \\
\hline 1991 & Guanarito virus \\
\hline 1989 & Hepatitis C \\
\hline 1988 & $\begin{array}{l}\text { Hepatitis E } \\
\text { Human herpesvirus 6 }\end{array}$ \\
\hline 1983 & HIV \\
\hline 1982 & Escherichia coli 0I57:H7 \\
\hline 1980 & Human T-lynphotropic virus \\
\hline 1977 & Campylobacter jejuni \\
\hline 1976 & $\begin{array}{l}\text { Cryptosporidium parvum } \\
\text { Legionnaires disease }\end{array}$ \\
\hline
\end{tabular}

Fonte: World Health Organisation 1999

A questi si vanno ad aggiungere quelli più recenti fra cui il Coronavirus della SARS che nel 20032004 ha creato molti problemi nei paesi asiatici e molto allarmismo anche nei nostri ed il recentissimo virus dell'influenza aviaria $\mathrm{H} 5 \mathrm{~N} 1$ che potrebbe diventare il responsabile di pandemia influenzale nell'uomo. L'HIV resta tuttavia l'esempio più importante di introduzione recente: si calcola che ci siano stati 22 milioni di morti nel mondo e che almeno 36 milioni di persone siano attualmente infettate.

Nuove acquisizioni legate agli sviluppi della ricerca

Lo sviluppo della ricerca ha consentito di documentare responsabilità microbiche anche nell'ambito di malattie croniche e degenerative. L'esempio più eclatante è indubbiamente Helicobacter pylori. La scoperta di un suo ruolo nelle patologie gastriche ha modificato radicalmente l'approccio diagnostico e terapeutico nella gestione dell'ulcera 
duodenale e delle patologie correlate. Gli ambiti della ricerca tutt'ora in corso, stanno ampliando ancora gli orizzonti e facendo intravedere come siano ancora molti gli aspetti da chiarire per quanto riguarda il vero ruolo dei microrganismi al di fuori della malattia infettiva acuta; a questo proposito la tabella 3 riassume alcune delle conoscenze attuali in proposito.

\section{Tabella 3.}

\begin{tabular}{ll}
\hline \multicolumn{2}{c}{ POSSIBILI CORRELAZIONI } \\
\hline $\begin{array}{ll}\text { Clamydia pneumoniae, Helicobacter pylori, } \\
\text { HSV, EBV, infezioni croniche peridontali }\end{array}$ & Malattie coronariche \\
\hline Mycobacterium avium subsp. paratuberculosis & Crohn's disease \\
\hline Propionibacterium acnes & Sciatica \\
\hline Yersinia spp., Salmonella spp., & \\
Campylobacter spp., Chlamiydia trachomatis & Artrite reattiva \\
\hline Klebsiella pneumoniae & Spondilite anchilosante \\
\hline Proteus mirabilis & Artrite reumatoide \\
\hline Micobatteri & Sarcoidosi \\
\hline Batteri responsabili di carie dentali & Endocarditi \\
\hline HPV & Carcinoma a cellule \\
& squamose della pelle \\
\hline Metabolici dei batteri anaerobi & in pazienti \\
\hline Schistosoma spp. & immunosoppressi \\
\hline Vaginosi batterica & Cancro del intestino \\
\hline
\end{tabular}

Oltre alle ricerche mirate anche studi su base epidemiologica correlano patologie croniche specifiche a cause infettive, come riportato in tabella 4.

\begin{tabular}{|c|c|}
\hline \multicolumn{2}{|c|}{$\begin{array}{c}\text { IPOTESI SU BASI EPIDEMIOLOGICHE } \\
\text { O EZIOLOGIA INFETTIVA }\end{array}$} \\
\hline Virus & Diabete giovanile \\
\hline eziologia infettiva & Artrite reumatoide \\
\hline Infezioni & Sclerosi multipla \\
\hline $\begin{array}{l}\text { Infezioni del bamb } \\
\text { dell'adolescente }\end{array}$ & Schizofrenia \\
\hline Infezioni & Leucemia del bambino \\
\hline Infezioni virali & Sindrome da fatica cronica \\
\hline
\end{tabular}

\section{Zoonosi}

Sono quattrocento le malattie che possono trasmettersi dagli animali all'uomo: un numero sicu- ramente elevato e probabilmente, anzi certamente, l'elenco non è ancora completo.

L'uomo condivide l'ambiente in cui vive con molte specie animali che a loro volta albergano un'ampia popolazione di microrganismi simbionti, saprofiti e parassiti in grado di passare all'uomo. Il potenziale infettivo è enorme, spesso sottostimato ed influenzato dai comportamenti umani stessi (es. nutrire animali erbivori con farine di derivazione animale) che alterano gli equilibri naturali. Infezioni zoonotiche trasmesse attraverso acqua e cibi contaminati coinvolgono frequentemente grosse fette di popolazione: uno degli esempi più eclatanti è quello dell'Escherichia coli O:157 che normalmente infetta i bovini. Questo microrganismo è in grado di produrre una tossina molto potente ed è stato causa di estese epidemie in Giappone, Canada e Scozia. Altro esempio è il Campylobacter, che scoperto nel 1977, è diventato la causa più frequente di enteriti in molte parti del mondo. Da ricordare ancora l'amebiasi e le infezioni da Yersinia e molte altre ancora.

\section{Bioterrorismo}

I recenti episodi di disseminazione di spore di antrace negli USA ha portato prepotentemente all'attenzione dei media il concetto che esiste la possibilità che, per motivi terroristici, vengano deliberatamente diffusi tra la popolazione microrganismi o loro derivati in grado di creare una consistente emergenza sanitaria che metterebbe facilmente in crisi il sistema di protezione civile seminando morte e panico. L'impatto dell'attività bioterroristica può essere limitabile in misura proporzionale al grado di preparazione del sistema sanitario nella velocità di identificazione dell'agente infettivo e nella messa in atto delle misure di contenimento ed isolamento più adeguate. In tabella 5 sono riportati gli agenti che possono essere utilizzati per tali scopi.

\section{Innovazioni tecnologiche ed organizzative}

Ai fattori sino a qui citati, relativi all'interazione uomo-microorganismi-ambiente che già di per sé dovrebbero essere il movente per rivedere ed

Tabella 5. Agenti biologici in grado di essere usati come armi

\begin{tabular}{llll}
\hline Batteri & Virus & Tossine & Rickettsia \\
\hline Bacillus anthracis & Encefalite equina venezulana & Botulino & Coxiella burnetti \\
\hline Yersinia pestis & Encefalite da morso di zecca & Ricino & (febbre Q) \\
\hline Francisella tularensis & Encefalite "Russian spring/summer” & Clostridium perfringens & R. prowazekii \\
\hline Brucella species & Febbre emorragica Crimea-Congo & Enterotossina B stafilococcica & \\
\hline Vibrio cholerae & Ebola & Micotossine & \\
\hline Burkholdeira pseudomallei & Marburg & & \\
\hline Burkholdeira mallei & Smallpox & & \\
\hline Salmonella typhi & Influenza & & \\
\hline
\end{tabular}

Fonte: Mesures for controllino the threat from biological weapons. London:The Royal Society, Luglio 2000 
aggiornare la rete di sorveglianza che deve garantire prevenzione e protezione adeguate a tutta la popolazione, si aggiungono aspetti tecnologici ed organizzativi che coinvolgono sia la diagnostica che l'organizzazione sanitaria.

\section{Impatto delle nuove tecnologie}

Le tecnologie sono diventate parte integrante della nostra vita quotidiana e se da un lato hanno migliorato il nostro modo di vivere, dall'altro hanno modificato e stanno modificando sempre di più i nostri rapporti con l'ambiente che ci circonda con conseguente apertura a nuovi rischi infettivi. L'aumento delle procedure invasive in ambito sanitario, come precedentemente citato, hanno comportato un aumento del rischio infettivo; l'uso di protesi, cateteri a permanenza e quant'altro ha fornito un ulteriore dimostrazione delle capacità adattative dei microrganismi che si inseriscono facilmente negli ambienti più disparati e si stabiliscono anche su superfici non organiche (13).

Un esempio di straordinaria rilevanza legato proprio al cambiamento della catena alimentare animale e quindi umana è la BSE (Bovine Spongiform Encephalopathy) e la malattia umana che sembra ad essa correlata, ovvero la Creutzfeld-Jacob Disease. Un altro esempio paradigmatico legato alla modifica degli ambienti in cui l'uomo vive è la malattia dei Legionari da Legionella pneumophila. È legata a certi tipi di condizionamento ambientale ed è ancora una volta la dimostrazione della grande capacità dei microrganismi di colonizzare ambienti nuovi.

\section{Innovazioni tecnologiche nella diagnostica}

I cambiamenti organizzativi legati alla modifica delle tecniche analitiche ed all'acquisizione di nuove strumentazioni sono probabilmente quelli che più facilmente si inseriscono nel laboratorio diagnostico. Le esperienze recenti in settori contigui ne sono una conferma. L'automazione spinta di estese branche della patologia clinica ha già determinato modifiche organizzative prima ancora di venir razionalmente accettate dagli operatori. Sino ad ora le modifiche hanno investito solo parzialmente la Microbiologia ma c'è chi vede imminente, come Ann Robinson ed altri (26), un modello di laboratorio che integra la Microbiologia direttamente nel "Core Laboratory", integrazione mediata da strumentazioni in grado di eseguire analisi automatizzate sul genoma e sugli elementi proteici microbici. La Robinson sottolinea però che ciò avrebbe conseguenze negative con perdita di qualità dovuta all'estensione dell'attività analitica a tecnici e laureati con una preparazione non specialistica. D'altra parte la spinta a diminuire $i$ tempi di rispo- sta (TAT) nel settore microbiologico, nonché la possibilità di usufruire di un servizio 24 ore al giorno, 7 giorni su 7 si fa sempre più pressante $\mathrm{e}$ l'aggancio al "Settore Urgenze" del laboratorio di patologia clinica è da più parti caldeggiato se non già attivato.

\section{Point of care testing (POCT)}

La pressione legata al contenimento dei costi ha favorito l'aumento dell'automazione, l'implementazione di sistemi informatici e la miniaturizzazione dei metodi analitici. A questo si è aggiunto, il tentativo recente di avvicinare maggiormente le attività mediche al paziente che per il laboratorio si traduce nel trasferimento dell'esecuzione di alcuni test presso il paziente, attuando quelli che vengono definiti dagli anglosassoni come POC (Point of care) (14). Il loro utilizzo è fonte di una continua e attenta riflessione (15) e non mancano dati a favore del fatto che l'utilizzo di alcuni test al di fuori dell'Ospedale può riflettersi in una diminuzione dell'accesso all'emergenza ed anche alla degenza nella struttura ospedaliera. Tutto questo tuttavia non sembra interessare il settore della diagnostica microbiologica per una serie di motivi che sono chiaramente deducibili dalla risposta che Robinson (26) ha dato alla domanda "I test al letto del paziente possono essere una alternativa clinicamente rilevante ed economicamente vantaggiosa rispetto a quelli eseguiti preso la Microbiologia?":

1. La complessità dei test microbiologici è attualmente incompatibile con la loro esecuzione all'esterno del laboratorio.

2. Il progresso nei metodi identificativi "non colturali" ed automatizzati potrà in futuro rendere disponibili test più sofisticati da poter eseguire in luoghi diversi dal laboratorio.

3. Attualmente i test decentralizzati che offrono TAT (tempi di attesa) rapidi risultano di scarsa affidabilità ed attendibilità sia per i limiti degli strumenti che per quelli dell'operatore e pertanto non sono in grado di rispondere alla domanda di salute attuale e futura.

Il progresso comporterà indubbiamente sviluppi ulteriori e cambiamenti nell'utilizzo del laboratorio con maggiore attenzione alla messa punto e diffusione di "home testing" e test da utilizzarsi presso $\mathrm{i}$ medici di medicina generale ma il tutto va visto nel contesto di quello che possiamo definire in generale come "Modifica dell'uso dell'Ospedale"

\section{Modifica dell'uso dell'ospedale}

La diminuzione dei tempi di degenza ospedaliera ed il conseguente spostamento dei pazienti in strutture diverse dall'Ospedale incidono sullo sviluppo delle infezioni e diventa pertanto imperati- 
vo pensare ad ampliare una delle attività fondamentali della Microbiologia che è quella di controllo delle infezioni ospedaliere, ponendo le basi per il "controllo delle infezioni comunitarie territoriali". L'importanza del collegamento laboratorio-territorio era già stigmatizzata in una non recente pubblicazione di Stainer dal significativo titolo "The virtual laboratory: regional clinical diagnostics for integrated delivery systems" dove si sostiene che "i laboratori sono chiamati a servire non solo i tradizionali pazienti dell'ospedale ma anche i medici del territorio distribuiti in cliniche, centri di chirurgia, medici di base in gruppo o singoli....."(27).

\section{Riduzione e/o contenimento dei costi}

Da alcuni anni la crescita dei costi del sistema sanitario nazionale è più elevata del PIL (Prodotto Interno Lordo) nazionale. Il finanziamento è pertanto sistematicamente inferiore al fabbisogno reale e da qui la necessità di attuare politiche di contenimento della spesa che non devono o dovrebbero diventare riduzione dei servizi per il cittadino. È quindi ovvio puntare sull'efficienza cercando di mantenere i livelli di assistenza a fronte di una diminuzione della spesa. La prima conseguenza dell'applicazione di questo concetto alla diagnostica in vitro è stata la tendenza alla concentrazione dei laboratori al fine di migliorarne l'efficienza con l'utilizzo razionale e completo delle tecnologie a disposizione e l'aumento del numero di esami per addetto. Questo però ha comportato problemi per l'efficacia dell'utilizzo del dato di laboratorio ed ha dimostrato che bisogna razionalizzare correttamente la spesa piuttosto che tagliarla in modo indiscriminato senza valutare le realtà delle situazioni e le logistiche specifiche. A questo proposito va citato il lavoro di Gazzaniga P. e Guglieri A., (11) realizzato per conto di Assobiomedica ed aggiornato al 2003 che sostiene quanto segue:

- sulla base dei dati disponibili non ci sono elementi per affermare che le risorse destinate alla diagnostica di laboratorio siano elevate rispetto a quelle mediamente destinatevi nei paesi OCSE;

- l'incidenza della spesa diagnostica sulla spesa sanitaria complessiva è generalmente stimata attorno all' $1 \%$, con range variabile dallo $0.6 \%$ della Gran Bretagna, all'1.6\% della Spagna (fonte: EDMA). In Italia si stima pari all'1.2\% (fonte: Assobiomedica);

- l'incidenza della spesa per la diagnostica di laboratorio sulla spesa sanitaria totale è sicuramente irrisoria e conseguentemente ancora più irrisorie devono considerarsi da un lato l'incidenza sulla stessa delle tecnologie diagnostiche (che rappresentano solo una parte della spesa diagnostica complessiva) e, dall'altro, la grandezza dei presunti risparmi ottenibili con tagli agli acquisti delle suddette tecnologie.

Perciò, dati i valori in campo, qualunque intervento non mirato all'eliminazione delle inefficienze di processo penalizza elementi sani, produce danni al sistema e non reca alcun significativo beneficio alla spesa.

Questa premessa consente di arrivare con corretta logica a sostenere che: “...gli investimenti nel settore della diagnostica, se ben programmati, possono portare a significativi risparmi per l'intero settore sanitario....". È evidente, infatti, che l'attenzione nella valutazione dei costi di laboratorio deve essere spostata dai bilanci dipartimentali verso il rapporto costo/beneficio valutando l'appropriatezza delle richieste analitiche attraverso una valutazione "dell'outcome" clinico (21).

\section{Efficienza ed efficacia del laboratorio}

Nel lavoro sopra citato, di Van Elder vengono riportati i risultati piuttosto inquietanti di un'indagine riguardanti l'utilizzo del laboratorio microbiologico per i pazienti con infezioni:

- il $60 \%$ ai pazienti ospedalizzati che avevano ricevuto un trattamento antibiotico non avevano avuto alcuna prescrizione per esame microbiologico;

- nella metà del 40\% dei casi rimanenti i clinici non conoscevano i risultati degli esami microbiologici che essi stessi avevano prescritto;

- nella metà del $20 \%$ dei casi residui di cui invece i risultati sono noti vengono seguite le raccomandazioni (trattamento antibiotico specifico).

Ne consegue che solo nel $10 \%$ dei pazienti trattati con antibiotici il dato del laboratorio microbiologico viene utilizzato.

Viene a questo punto spontaneo chiedersi come aumentare l'impatto clinico della Microbiologia. I punti su cui poter agire sono essenzialmente due:

1. rilevanza clinica delle risposte;

2. rapidità delle risposte.

A proposito del secondo punto Doern et al. (6) in "Clinical impact of rapid in vitro susceptibility testing and bacterial identification" dimostrano che il costo finale di un paziente nella struttura, la morbilità e mortalità risultano significativamente più bassi quando le risposte pervengono in tempi rapidi.

\section{Strutturazione équipe e clima interno}

Un altro aspetto tutt'altro che marginale da tener presente quando si affronta il tema del cambiamento è la situazione del personale in numero, età e motivazione.

L'ambiente di lavoro risente sicuramente della 
tipologia e qualità di lavoro eseguito, del clima interno e delle prospettive professionali di coloro che vi lavorano. Alcuni recenti lavori di Ehrardt, Taggard, Pitt e Sands $(8,28,23)$ evidenziano che negli Stati uniti c'è una vera e propria crisi di vocazioni per il lavoro in laboratorio in generale ed in Microbiologia in particolare. L'età media dei Microbiologi si aggira sui 50 anni, mancano operatori delle fasce di età più giovani che hanno maggiori affinità all'utilizzo dei computer e delle biotecnologie. Una età media equivalente è stata evidenziata anche in Italia da un recente studio della FeSIM (Federazione Società Italiane di Microbiologia) (4) sulla situazione dei laboratori di Microbiologia ed è certo, anche se non scientificamente misurato, che il blocco ormai decennale del turn-over in sanità e l'idea che la tecnologia in laboratorio possa ridurre la necessità di personale senza fare i dovuti distinguo, comporta in Italia le stesse difficoltà. D'altra parte, come sottolinea bene Ehrardt, è in discussione anche l'idea che più è grande il laboratorio, più test si fanno, migliore è il risultato: il laboratorio migliore non è il più grande ma il più efficiente nel salvaguardare la salute della popolazione nel suo raggio di azione. All'efficienza vera si arriva intervenendo in primis sull'appropriatezza collegata alle risorse interne e alle necessità reali della comunità che utilizza il laboratorio.

Il clima interno e quella che viene chiamata "motivazione dell'équipe" sono per Pitt e Sands un altro aspetto da affrontare: équipes impegnate in un miglioramento continuo della qualità, in gruppi non superiori alle 30 persone costituirebbero il miglior aiuto per il mantenimento di un buon clima interno.

\section{MODELLI ORGANIZZATIVI}

L'analisi sino a qui fatta ha preso in considerazione le motivazioni per cui è necessario un cambiamento e le numerose variabili che lo condizionano. A questo punto ci si chiede cosa e come fare per realizzarlo in modo corretto ed arrivare ad una organizzazione razionale che renda il nostro operare quotidiano efficiente ed efficace.

In un articolo redatto a cura della Società Americana di Malattie Infettive, Peterson et al. (20) evidenzia come nel recente passato le misure di contenimento dei costi abbiano portato negli Stati Uniti a:

- ristrutturare;

- centralizzare;

- consolidare.

Sono stati istituiti servizi di laboratorio che includono anche i laboratori di microbiologia mediante la creazione di grossi centri in grado di servire più ospedali e la concomitante diminuzione delle figure professionali dedicate alla microbiologia. Secondo un'intervista fatta ai Direttori di questi servizi $\mathrm{i}$ vantaggi della centralizzazione sarebbero:

1. riduzione dei costi;

2. aumentata accuratezza delle analisi poco frequenti come conseguenza dell'incremento del numero;

3. aumento dell'offerta della tipologia delle analisi come risultato dell' aumento del bacino d'utenza;

4. aumento della standardizzazione delle metodiche;

5. aumento della possibilità di formazione sia verso l'interno che verso l'esterno.

In realtà questa centralizzazione è caratterizzata anche da una serie di svantaggi:

1. scarsa comunicazione tra medici di reparto e personale di laboratorio;

2. problemi ricorrenti nel trasporto dei campioni;

3. problemi interpretativi dell'esame diretto (Gram) dovuto all'utilizzo di laureati non specialisti nei laboratori dedicati alle urgenze;

4. difficoltà di controllo delle infezioni ospedaliere dovuta a perdita di interazione del microbiologo con lo staff medico/infermieristico dell'ospedale.

Peterson perciò conclude sostenendo che le difficoltà di mantenimento della qualità verificate tramite l'abbassamento delle performances nelle "proficiency surveys", la difficoltà di raccolta ed invio dei campioni che incidono in una fase fondamentale dell'analisi del campione microbiologico che è quella preanalitica, il rarefarsi delle comunicazioni tra il reparto ed il microbiologo, nonché la difficoltà di svolgere una corretta didattica per i nuovi microbiologi, pone la centralizzazione dei servizi di microbiologia in un'ottica negativa nei confronti dei fabbisogni della salute dei cittadini.

Per quanto riguarda l'Europa, il Ministero della Salute inglese ha stato stilato un documento, unico nel suo genere, per completezza dell'analisi dei bisogni e delle priorità, il cui titolo è "Getting ahead of the curve" (2) e che definisce le linee organizzative per rispondere alle nuove necessità in tema di malattie infettive. In questo contesto viene anche definita l'organizzazione del Servizio di Microbiologia che proprio nella sorveglianza delle malattie infettive vede uno dei punti principali della propria "mission" e che deve dare uguale enfasi ed importanza sia al ruolo diagnostico che lo caratterizza, sia al ruolo di "protezione pubblica". Un moderno sistema per combattere le malattie infettive ed avere una più ampia protezione della salute della popolazione deve, secondo il documento, basarsi essenzial- 
mente su due punti:

- riconoscimento precoce dell'infezione e notifica tempestiva alle autorità competenti in base ad una accurata diagnosi sia clinica che di laboratorio;

- un forte sistema di sorveglianza supportato dai servizi di microbiologia sia diagnostici che di riferimento (accreditati).

Nel nostro paese in realtà al Servizio di Microbiologia viene riconosciuto prevalentemente il ruolo di diagnostica clinica piuttosto che quello di sorveglianza, mentre il grado di importanza dei due ruoli dovrebbe essere lo stesso. Nel documento inglese oltre a rimarcare la duplicità dei ruoli viene anche affermato che la raccolta e diffusione dei dati epidemiologici deve diventare obbligatoria per i servizi microbiologici in quanto cardine del sistema di controllo.

In "Scenari per il settore della diagnostica in vitro" Gazzaniga e Guglieri nel 2003 (11), sottolineavano che anche in Italia le pressioni riconducibili da un lato alle crescenti opportunità offerte dall'evoluzione delle tecnologie diagnostiche in vitro e, dall'altro alle crescenti difficoltà di bilancio pubblico degli ultimi 5 anni, hanno dato forza ad un generale processo di consolidamento dei laboratori. Per quanto riguarda i laboratori pubblici la forza motrice di questo processo è data dalla necessità di abbassare $\mathrm{i}$ costi di gestione dei servizi sanitari, nessuno escluso.

Ciò, attraverso l'aziendalizzazione delle strutture sanitarie, ha portato all'accorpamento delle medesime e la conseguente spinta alla riorganizzazione dei servizi, tra cui i servizi di laboratorio, che in più casi sono stati accorpati in un processo di accentramento forzato.

Nel tentativo di dare un modello a queste riorganizzazioni ci si è riferiti alla letteratura americana che, negli anni 90, proponeva sostanzialmente due tipologie organizzative "hub and spoke" $\mathrm{e}$ "core lab".

Il primo caratterizzato da un grande laboratorio centrale totalmente automatizzato con una serie di piccoli laboratori satellite (nelle strutture ospedaliere) dove eseguire gli esami urgenti e tutti quelli che si decide di non centralizzare.

Il secondo è un laboratorio che per dimensioni è una via di mezzo tra un Hub ed uno Spoke, organizzato con una automazione ad isole ed in grado di lavorare con TAT corti ( 2 ore) così da assicurare con le medesime macchine sia una attività di routine che le urgenze (10).

Alcune esperienze sono state tentate anche in Italia, con valutazioni alterne. Secondo le Società Scientifiche di Laboratorio organizzatrici del Convegno "La medicina di laboratorio in Italia: come disegnare il futuro" tenutosi a Milano il 10-
11 Novembre 2003, la criticità principale emersa a carico delle soluzioni adottate in Italia, è quella di privilegiare le considerazioni economiche rispetto a quelle "scientifiche".

Secondo il documento inglese "Modernizing pathology service" (3) una riorganizzazione dovrebbe basarsi su tre punti fondamentali:

- progettare il servizio sulla base dei bisogni del paziente e delle realtà locali;

- fornire risposte tempestive e qualitativamente ottimali;

- supportare la buona pratica clinica in modo da assicurare esiti favorevoli per i pazienti ed il sistema sanitario.

Oltre a tutto questo va tenuto presente, a nostro parere, quanto riportato da Reller e Weinstein (24) in merito alla mission dei Laboratori di Microbiologia:

1. i dati generati dal laboratorio di microbiologia sono critici per la diagnosi e la cura delle malattie infettive;

2. il laboratorio di microbiologia è la spina dorsale del controllo infettivo nei sistemi sanitari, provvede ad una informazione cruciale sulle infezioni comunitarie riguardante la prevalenza degli agenti infettivi e la loro sensibilità agli antibiotici;

3. in partnership con il dipartimento di prevenzione, rappresenta la prima linea di difesa contro le emergenze infettive, resistenza agli antibiotici e bioterrorismo inclusi;

4. è un riferimento per la formazione in campo infettivo.

Gli autori mettono anche in guardia dalla eccessiva spinta alla centralizzazione della microbiologia, sottolineando che se i laboratori sono localizzati a forte distanza dai pazienti e dai loro medici si può avere compromissione:

- dei campioni che contengono microrganismi fragili, a causa dei tempi di trasporto;

- della qualità analitica;

- della comunicazione tra laboratorio e reparti di degenza e non;

- dell'utilizzo delle risposte (anche tempestive).

Dalla valutazione di quanto discusso appare evidente che per poter operare correttamente non possiamo prescindere dalla definizione di un piano per la microbiologia clinica, su base nazionale che possa rispondere a livelli di attività diversi e, che possa essere recepito in maniera organica nelle singole realtà regionali.

L'organizzazione della microbiologia a livello regionale non può comunque prescindere dalle singole realtà territoriali: è solo attraverso un'attenta, meditata ed equilibrata conoscenza e riorganizzazione dell'esistente che si può affrontare razionalmente il cambiamento. 


\section{UNA PROPOSTA ORGANIZZATIVA}

Tenendo presente che obbiettivi fondamentali della Microbiologia clinica sono:

- diagnosi di laboratorio delle malattie infettive

- rilevamento delle resistenze ai farmaci antimicrobici quale strumento da fornire al clinico per un trattamento mirato

- controllo della diffusione di infezioni nelle strutture sanitarie (inf. ospedaliere ecc.)

- costituire la prima linea di difesa per le emergenze infettive e per il bioterrorismo, in collaborazione con il Dipartimento di prevenzione

- essere riferimento per la formazione specifica i principi su cui si deve basare la riorganizzazione, come ampiamente sottolineato nel documento (Getting Ahead the Curve. Department of Health UK), sono essenzialmente tre:

1. configurare il servizio di microbiologia sulla base degli effettivi bisogni della popolazione locale

2. fornire risposte in tempo utile e qualitativamente ottimali

3. supportare la "buona pratica clinica" in modo da assicurare risposte adeguate per i pazienti e costi accettabili per il sistema sanitario regionale (SSR).

Sulla base di esperienze già consolidate è emerso però che uno dei fattori che condizionano le scelte nella distribuzione territoriale dei servizi è quello della "distanza" dai punti di raccolta dei campioni biologici. Infatti il trasporto può compromettere la vitalità di microrganismi fragili o l'integrità dei loro componenti, nonché il rapporto delle popolazioni microbiche.

Inoltre le comunicazioni tra laboratorio e medici curanti diventano più difficoltose con la distanza e diminuisce l'efficacia della tempestività della risposta. La soluzione migliore di questo problema è un corretto equilibrio tra accentramento delle attività diagnostiche e "distanza".

Per poter soddisfare questi principi, tenendo presente i costi delle nuove tecnologie e la necessità di esperienze professionali specifiche degli operatori (che si acquisiscono e si mantengono con una attività continua su un adeguato numero di campioni) la logica dell'accentramento sembra la soluzione più ovvia ma è la meno adatta.

\section{Il Dipartimento di Microbiologia (struttura ed organizzazione)}

Sulla base delle premesse e di quanto emerge da esperienze nazionali ed internazionali l'organizzazione della microbiologia dovrebbe prevedere un Dipartimento di Microbiologia, strutturato su base provinciale o area vasta e costituito da una "struttura complessa" centrale e da strutture peri- feriche (anche semplici) e così articolato:

- Laboratorio presso l'azienda ospedaliera principale (struttura complessa), diretto da uno specialista in Microbiologia e Virologia e suddiviso nei settori che contraddistinguono la Microbiologia ossia batteriologia - micologia parassitologia - virologia - sierologia. Per ognuno di questi settori, se attivato, dovrebbe esserci almeno un laureato specialista.

- Strutture periferiche di Microbiologia, dislocate nell'ambito dei laboratori generali periferici ma afferenti al Dipartimento del quale è parte integrante.

Il Dipartimento di Microbiologia deve integrarsi attivamente con il Dipartimento di Prevenzione per quanto riguarda la dinamica delle malattie infettive sul territorio, costituendo la prima linea di difesa contro le emergenze infettive, cardine importante nella "mission" della microbiologia.

Parallelamente va perseguita una interazione attiva con le strutture di ricerca (IRCCS ed Università) presenti sul territorio in modo da implementare in modo coordinato le linee di ricerca e la loro ricaduta sulla diagnostica.

\section{I centri di Riferimento}

Tenendo presente che la qualità delle prestazioni diagnostiche è strettamente legata all'esperienza professionale degli operatori, che si acquisisce, consolida e mantiene con una attività continua su di un adeguato numero di campioni, è opportuno centralizzare alcune procedure diagnostiche tramite l'identificazione di centri di riferimento regionali. La centralizzazione è anche la soluzione più razionale per ottimizzare i costi delle nuove tecnologie e per gestire adeguatamente il problema della garanzia di qualità. I centri di riferimento vanno identificati per tipologia della patologia o di microrganismi specifici (es: centro per la resistenza ai farmaci antibatterici; centro per la diagnostica delle infezioni da micobatteri; centro per la diagnostica delle malattie trasmesse da zecche; centro per la diagnostica degli enterobatteri patogeni; centro per la farmacoresistenza di HIV e fenotipizzazione virale; centro per la diagnostica avanzata delle epatiti; centro per la diagnostica di legionellosi, ecc) e possono essere distribuiti sia presso le strutture complesse che quelle semplici. È evidente la necessità di tener in considerazione gli standars prestazionali minimi da raggiungere, pur tuttavia è comunque indispensabile centralizzare gli esami poco frequenti. Le singole realtà territoriali, attività specifiche consolidate, tecnologie disponibili devono far parte dei criteri da seguire per la loro identificazione.

L'organizzazione di un efficiente sistema di tra- 
sporto sia su base regionale che dipartimentale, ed il collegamento informatico di questi centri, e la gestione delle informazioni epidemiologiche da essi prodotte, sono requisiti aggiuntivi, ma altrettanto importanti per supportare tutta l'organizzazione.

\section{Il comitato regionale di coordinamento}

In un network regionale così delineato è importante prevedere una struttura di coordinamento, poiché sarà necessario attivare il massimo livello possibile di comunicazione, scambio di informazioni, pianificazione delle attività, risoluzione concordata dei problemi. Oltre a ciò si ritiene necessario un organo in grado di attivare prontamente il ruolo di sentinella rispetto ad emergenze infettive. Sarebbe opportuno che questo comitato fosse composto almeno dai responsabili dei dipartimenti di Microbiologia regionali. Un ruolo nella partecipazione al coordinamento potrebbe essere esteso ai Dipartimenti di prevenzione, ai centri di ricerca Universitari, agli IRCCS ed agli Infettivologi.

\section{BIBLIOGRAFIA}

1. Andreu A. Medical microbiology in the european union. Enferm Infec Microbiol Clin 2003; 21 supl 2: $9-11$.

2. A.A.V.V. Getting ahead of the curve. A strategy for combating infectious diseases (including other aspects of healt protection. Department of Health; United Kingdom 2002).

3. A.A. V.V. Modernising pathology services Department of Health; United Kingdom 2004

4. Bassi M, Falcitelli N. Strutture di diagnostica microbiologica in Italia. Ed. Fondazione Smith Kline Roma Dic 2004

5. Daniels MJ, Hitchings MR, Greig A. The risk of disease transmission to livestock posed by contamination of farm stored feed by wildlife excreta. Epidemiol Infect 2003; 130 (3): 561-8.

6. Doern GV, Vautour R, Gaudet M, Levy B. Clinical impact of rapid in vitro susceptibility testing and bacterial identification. J Clin Microbiol 1994; 32: 1757-62.

7. Dunne Jr WM, Pinkard JK, Hooper LV. Clinical Microbiology in the Year 2025. J Clin Microbiol 2002; 40: 3889-93.

8. Ehrhardt P. Surviving the staffing crisis. Clin Leadersh Manag Rev 2002 Nov-Dec; 16(6): 412-4.

9. Gazzaniga P, Guglieri A. La spesa sanitaria, la diagnostica di laboratorio e il mercato delle tecnologie. Terzo aggiornamento. CSA Milano Ottobre 2000; N. 8.

10. Gazzaniga P. L'impatto economico dell'evoluzione tecnologica: aspetti di valutazione. CSA Milano Febbraio 1999; N. 5.

11. Gazzaniga P, Guglieri A. Scenari per il settore della diagnostica in vitro. CSA Milano Novembre 2003; N. 10.

12. Haas WH, Breuer T, Pfaff G, et al. Imported Lassa fever in Germany: surveilance and management of contact persons. Clin Infect Dis 2003; 36: 1254-8.

13. Hanssen AD. The diagnosis of prosthetic joint infec- tion: current techniques and emerging technologies. Clin Orthop Relat Res 2005; 437: 55-8.

14. Kasahara Y, Ashihara Y. Simple devices and their possible application in clinical laboratory downsizing. Clin Chim Acta 1997; 267: 87-102.

15. Lehmann CA. The future of home testing Implication for traditional laboratories. Clinica Chimica Acta 2002; 323: 31-6.

16. Lick SD, Edozie SN, Woodside KJ, Conti VR. Streptococcus viridans endocarditis from tongue piercing. J Emerg Med 2005; 29: 57-9.

17. Masin LJ. Where are we going? Health-care professionals and the staffing crisis. Clin Leadersh Manag Rev 2003 Sep-Oct; 17(5): 293-6.

18. Mertens TE, Low-Beer D. The newest epidemic: a review of HIV/AIDS in Central and Eastern Europe Int J STD AIDS 2003; 14: 361-71.

19. Paxton A. Straight talk from core lab survivors. www.cap.org September 2000.

20. Peterson LR, Brossette SE. Hunting Health CareAssociated Infections from the Clinical Microbiology Laboratory: Passive, Active, and Virtual Surveillance. J Clin Microbiol 2002; 40: 1-4.

21. Peterson RL, Hamilton JD, Baron EJ, et al. Role of clinical microbiology laboratories in the management and control of infectious diseases and the delivery of health care. Clinl Infect Dis 2001; 32: 605-11.

22. Petrak RM, Sexton DJ, Butera ML, Tenenbaum MJ, et al. The value of an infectious specialist. Clin Infect Dis 2003; 36: 1013-7.

23. Pitt SJ, Sands RL. Effect of staff attitudes on quality in clinical microbiology services. Br J Biomed Sci. 2002; 59: 69-75.

24. Reller LB, Weinstein MP. Policy statement on consolidation of clinical microbiology laboratories. Clin Infec Dis 2001; 32: 64-5.

25. Rigoli E, Rota A. L'organizzazione dei servizi di Microbiologia. Edizioni AMCLI Ott. 1990 Treviso.

26. Robinson A, Marcon M, Mortensen JE, et al. Controversies affecting the future practice of clinical microbiology. J Clin Microbiol 1999; 37: 883-9.

27. Stainer JW. The virtual laboratory: Regional clinical diagnostics for integrated delivery systems. Healthcare Financial Management 1997; 51 (11): 40-4.

28. Taggart LM. The laboratory manager's new role as retention officer. Clin Leadersh Manag Rev 2002 Nov-Dec; 16(6): 415-8.

29. Van Eldere J. Models for change in clinical microbiology. Clinical microbiology and infection 2000; 8: 445-8.

30. Wheater CP, Cook PA, Clark P, Sved Q, Bellis MA. Re-emerging syphilis: a detrended correspondence analysis of a behaviour of HIV positive and negative gay man. BMC Public Health 2003; 29: 30-4.

\section{ALTRE LETTURE UTILI}

- Baron EJ. Speculation on the microbiology laboratory of the future. Clin Infect Dis 2002; 35: S 84-7.

- Bille J. Clinical microbiology - the model of University Hospital of Lausanne, Switzerland. CMI 2000; 6: 405-7.

- Gilbert GL. Molecular diagnostics in infectious diseases and public health microbiology: cottage industry to postgenomics. Trends in molecular medicine 2002;8 (6): 280-7.

- Isemberg HD. Clinical microbiology: past, present, and future. J Clin Microbiol 2003; 41: 917-9.

- Madeley CR. The future of diagnostic virology, part 2: 
staffing the service. CMI 2000; 6: 342-7.

- Nicolas-Chanoine MH. Future needs - diagnostic services. CMI 2000; 6: 423-5.

- Peddecord KM, Baron EJ, Frencis D, Drew JA. Quality perceptions of Mycrobiology services. A survey of infectiuos diseases specialists. Am J Clin Pathol 1996; 105: 58-64.

- Roberts C. Training in clinical microbiology in UK. CMI 2000; 6: 435-7.

- Scott GM. Clinical microbiology - the UK model. CMI 2000; 6: 402-4.

- Van der Meer JWM. Future needs - clinical services for infectious diseases. CMI 2000; 6: 426-7.

\section{Giorgio Mucignat}

Struttura Operativa Semplice di

Microbiologia Clinica e T. A.

Dipartimento di Medicina di Laboratorio Azienda Ospedaliera

"Santa Maria degli Angeli" Pordenone

Via Montereale, 24 - 33170 Pordenone

Tel. 0434399648 Fax 0434399685

E-mail: giorgio.mucignat@aopn.fvg.it 